\title{
Subminiature eddy current transducers for studying aluminium-dielectric and steel-dielectric junctions
}

\author{
Dmitriev Sergey, Malikov Vladimir, Sagalakov \\ Anatoly, Katasonov Alexander \\ Faculty of physics and technology \\ Altai State University, ASU \\ Barnaul, Russia \\ dmitrsf@gmail.com
}

\author{
Ishkov Alexey \\ Faculty of physics and technology \\ Altai State Agrarian University, ASAU \\ Barnaul, Russia \\ mirotnas@gmail.com
}

\begin{abstract}
Based on a eddy current transducer (ECT), a probe has been designed to research metal-dielectric structures (MDS). A scheme that uses a computer as a generator and receiver of signals from windings is proposed. The measurement procedure allowing one to detect defects in laminate composites with a high accuracy is described. The transducer was tested on the layered structure consisting of paper and aluminum layers with a thickness of $100 \mu \mathrm{m}$ each in which the model defect was placed. The dependences of the ECT signal on the defect in this structure are given. A hardware-software complex was built to perform experimental studies of steel at the interface boundary. Test results are reported for a specimen with continuous and discrete measurements taken at different frequencies. The curve of introduced voltage amplitude as a function of sensor location was constructed to study in detail the introduced voltage drop at the ferromagnetic-dielectric interface.
\end{abstract}

Keywords-eddy-current transducer; electrical steel; introduced voltage; measuring system; metal-dielectric structures

\section{INTRODUCTION}

A subminiature eddy-current transducer has been designed to monitor physical parameters when studying the properties of metal-dielectric junctions. The developed measuring system allows effective investigate the metal-insulator transitions in miniature metal-polymer composite objects. Similar composites may include several metal layers separated by thin dielectric layers of polymer. The typical defects of such materials are, for example, the disturbance of layers continuity and the formation of link between the layers.

Defects inspection of MDS is performed to determine the following standard defects: defect of the metallic and (or) polymer layer uniformity, the number and thickness of the layers, conducting and non-conducting strips between layers, deformation of the metal layer surface, change in the state of boundary between the metal and polymer layers of the MDS. In this case, one of the universal parameters sensitive to all the listed defects of MDS is the local electrical conductivity of the material and its distribution over the surface, associated with the topology of the electromagnetic field in the material interacting with these defects $[1,2]$.

According to the literature review, there are many types of inspections to evaluate composites and there are many proposed methods by researchers for each of which. For damage identification in aircraft composite structures, aircraft composites assessment, and health monitoring of aerospace composite structures the suggested methods in the literature is ultrasonic testing [3, 4], thermographic testing [5, 6], vibration methods [7, 8], infrared thermography [9], shearography [10], and XCT [11].

Ultrasonic testing is the most applied method in health monitoring of a composite wing-box structure [12], damage identification in aircraft composite structures [13], aircraft composites assessment [10], health monitoring of aerospace composite structures [14], and structural health monitoring (SHM) [4]

Pulse echo ultrasonic method can readily locate defects in homogeneous materials. In this method, the operator more concerns about the transit time of the wave and the energy loss due to attenuation and wave scattering on flaws. It helps to locate inconsistency in a material whether it is homogeneous or heterogeneous [15]. For large defect detection, location, and imaging purposes, and quality control, ultrasonic pulse velocity measurements are quite suitable [16].

Electromagnetic Testing (ET) methods use magnetism and electricity to detect and evaluate fractures, faults, corrosion or other conditions of materials. ET induces electric currents, magnetic fields, or both inside a test object and observes the electromagnetic response. Electromagnetic (EM) methods include Eddy Current Testing (EC) [17], Remote Field Testing, Magnetic Flux Leakage and Alternating Current Field Measurement. In each of these techniques, the underlying physics is fundamentally different as the fields described by different classes of partial differential equations [18].

The eddy-current method (ECM) is one of the main methods to inspect defects for the purpose to control various materials and products in modern technology.

A qualitative and reliable assessment of the technical condition of hazardous production facilities is an important task.

Over the past century, many non-destructive methods have been studied in research on fatigue damage evaluation. Some of the methods that showed promise for metals and alloys 
included x-ray diffraction, see e.g., laser diffraction, infrared thermography and positron annihilation [19].

The method of eddy current nondestructive testing is widely used for the control of steel products, based on the analysis of electromagnetic field interaction with eddy currents caused by this field [20].

Thus, in works [21, 22], a method for evaluation of brittle fracture in metal equipment by eddy current testing with the help of proximity and overhead sensors is proposed. 09G2S low-alloy steel was used for experiments.

In the course of this study, the task was to demonstrate the possibility to scan steel-insulator transitions with a developed eddy-current transducer to study the steel surface characteristics.

The eddy-current control method is often used for evaluation of various steel parameters. The investigations [23, 24] demonstrated practicality of using such method for fault location in AISI 304 steels. At the same time, the developed gage positioning system with the pitch of $0.1 \mathrm{~mm}$ was used for accurate gage movement. The works $[25,26]$ highlight that one of the obvious advantages of using the eddy-current method for investigation of conductive materials is its applicability for diagnostic of materials with paint and varnish, and other non-conductive coatings.

The aim of the study was to develop, research, optimize and test the developed ECT and obtain mathematical dependencies describing the change in a signal value at ECT when various properties of studied steels and aluminium and transducer parameters change.

\section{MATERIALS AND MEASUREMENT PROCEDURE}

Subminiature ECT $[27,28]$ is designed for experimentally local studies of the thickness of various coatings and to determine the effect of various coatings on the output signal value. The developed subminiature ECT represents a core wrapped with the following windings: energizing, measuring and compensation. ECT consists of a core wrapped with the energizing, measuring and compensation windings. Both the windings and the core are impregnated with a compound. They are enclosed in a washer of corundum. This equates to an increase of the mechanical stability of the transducer.

To test different conductive materials, a developed transducer is used, which is connected to a personal computer via a sound card that is used as a generator and as a signal transducer. The signal thus is sent directly to the energizing winding.

The software is able to control the quantity of a signal applied to the energizing winding and also allows to read the voltage values from the measuring winding, which, taking into account the calibration, are converted into conductivity values. The developed software allows measuring the thickness of conductive and dielectric non-ferromagnetic coatings and conductive materials

ECT winding coils consist of a copper wire with the thickness of $5 \mu \mathrm{m}$. The core is made of ferrite 2000 NM3 with an initial magnetic permittivity value of 2000 and has a pyramidic shape. Characteristics of the developed transducer make it possible to achieve high localization of the control, namely, to localize the field within $2500 \mu \mathrm{m}^{2}$. The developed system provides a significant depth of penetration of the field into the prototype system up to values of $\sim 5 \mathrm{~mm}$ (at frequencies of $500 \mathrm{~Hz}$.)

The eddy-current transducer (Figure 1) is a transformer with measuring (1), exciting (2), and compensation (3) windings and a magnetic circuit 4 , which is located inside the cylindrical platform 5 with tracks that are cut on the external side for windings. The platform is impregnated with a compound 6 at a temperature of $200^{\circ} \mathrm{C}$ to prevent the disintegration of the windings when the ferrite screen 7 , which is intended for the localization of the electromagnetic field on the tested object, is put in place. From the outside, the transducer is contained in a corundum washer 8, which protects the core 4 from contacting the tested object.

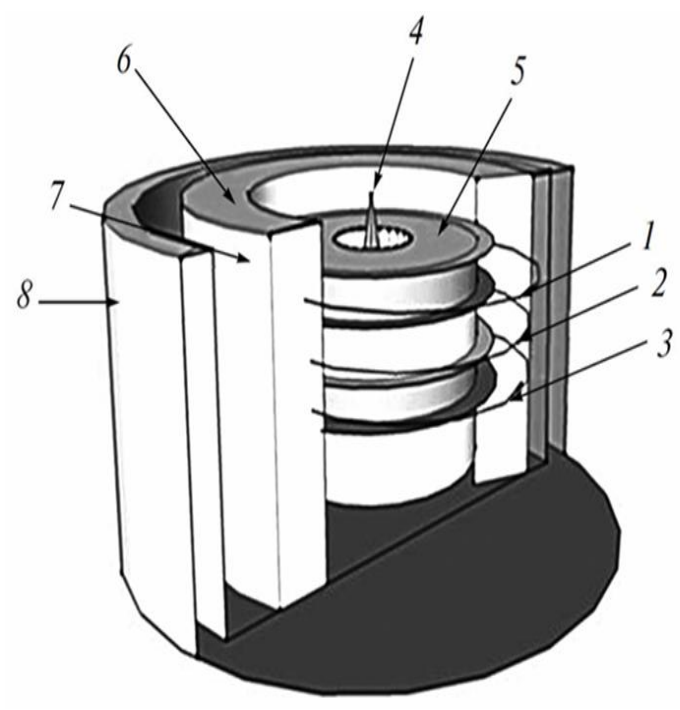

Fig. 1. Scheme of eddy current transducer

The software coded in $\mathrm{C}++$ for Windows allows controlling the signal on the energizing winding and receiving the signal from the measuring winding. With the help of the software it is possible to effectively control the signal, which is applied directly to the energizing winding. Also with this software it is possible to receive a signal directly from the measuring winding. The impressed voltage can be controlled using a special mixer built into the Windows. With the help of this mixer, the frequency and amplitude parameters of the generator sinusoidal signal are set. In turn, the sound card makes it possible to extend the signal bandwidth, which is applied directly to the energizing winding.

\section{RESULTS AND DISCUSSION}

\section{A. Scanning the layered structure aluminium-paper}

Experiments. To demonstrate the proposed method operability, the structure of alternating aluminum foil of 100 
$\mu \mathrm{m}$ thick and paper of $100 \mu \mathrm{m}$ thick has been used. As a defect model between the layers a hollow parallelepiped with a wall of $300 \mu \mathrm{m}$ thick has been placed. The defect was at a distance of $600 \mu \mathrm{m}$ from the sensor in the depth of the layered structure.

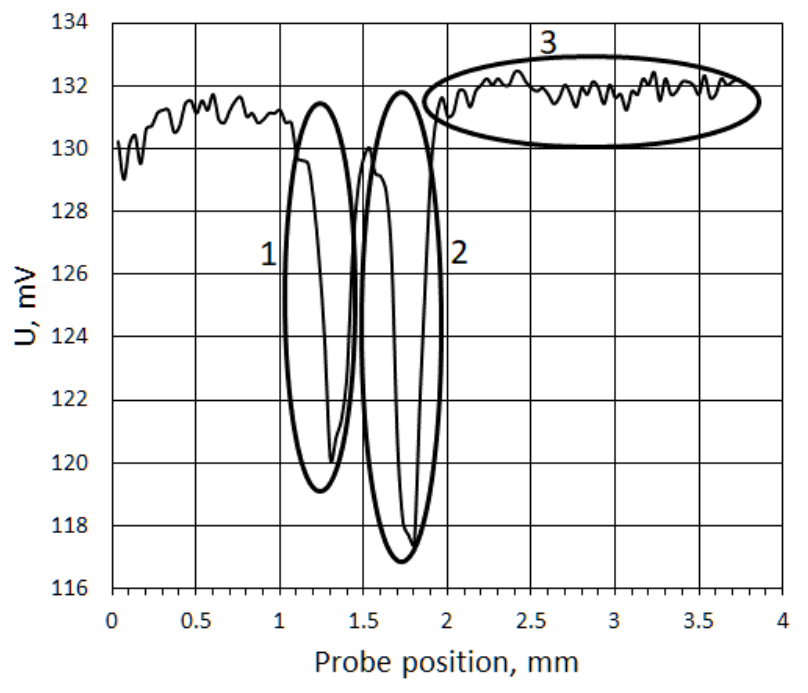

Fig. 2. A pattern observed when the probe moves along the layered medium with a defect. The frequency of the transducer is $1000 \mathrm{~Hz}:(1,2)$ walls of the parallelepiped, (3) defectless part of the sample

Figure 2 shows the picture observed when the probe moves above the layered medium inside which a defect is located. The signal level from the measuring winding characterizes conductivity values on the survey section. For basic operating frequency of $1000 \mathrm{~Hz}$ the voltage level included in the measuring winding was $130 \pm 2 \mathrm{mV}$. Areas 1 and 2 on the graph where in voltage level drop is up to $115 \mathrm{mV}$ correspond to the walls of the parallelepiped. This change in the signal amplitude is equal to $11 \%$ of the signal level, corresponding to the defectless section of the sample. At the same time the fluctuations of signal amplitude at the defectless section does not exceed $4 \mathrm{mV}$, representing $3 \%$ of the signal corresponding to the defectless region of the sample.

From the graph shown in Fig. 3.,4. clearly visible change in amplitude when testing the transducer at other frequencies. These changes are caused by the increase in frequency at condition less penetration depth of the eddy currents in the depth of the layered structure and increase in the influence of different small cracks on the surface of the layered structure, as well as external influences.

At a frequency of measurement equal to $6000 \mathrm{~Hz}$ (Fig. 3), the defect model is still clearly visible, however the amplitude of oscillation on a defectless portion of the sample, already exceed $7 \%$ of the signal level corresponding to the defectless portion of the sample.

In laboratory and industrial measurements, such oscillation amplitude can be caused by external influences, unconditioned the presence of defects or small defects on the surface of the metal layer. In the study of the test object with unknown defects, similar changes amplitude may mistakenly be interpreted as a defect.

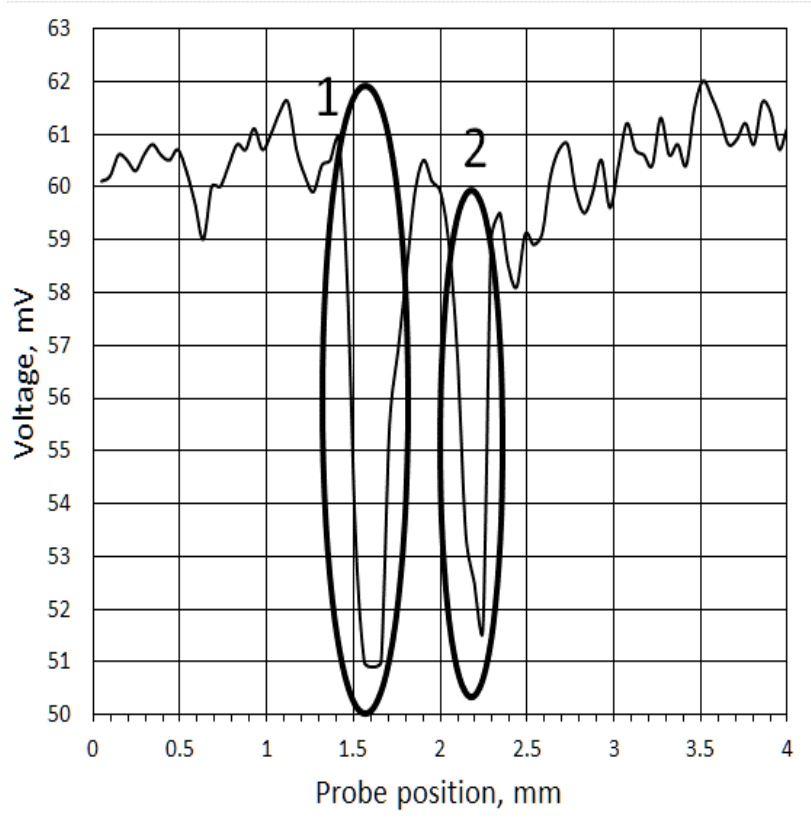

Fig. 3. A pattern observed when the transducer moves along the layered medium with a defect. The frequency of the transducer is $6000 \mathrm{~Hz}$

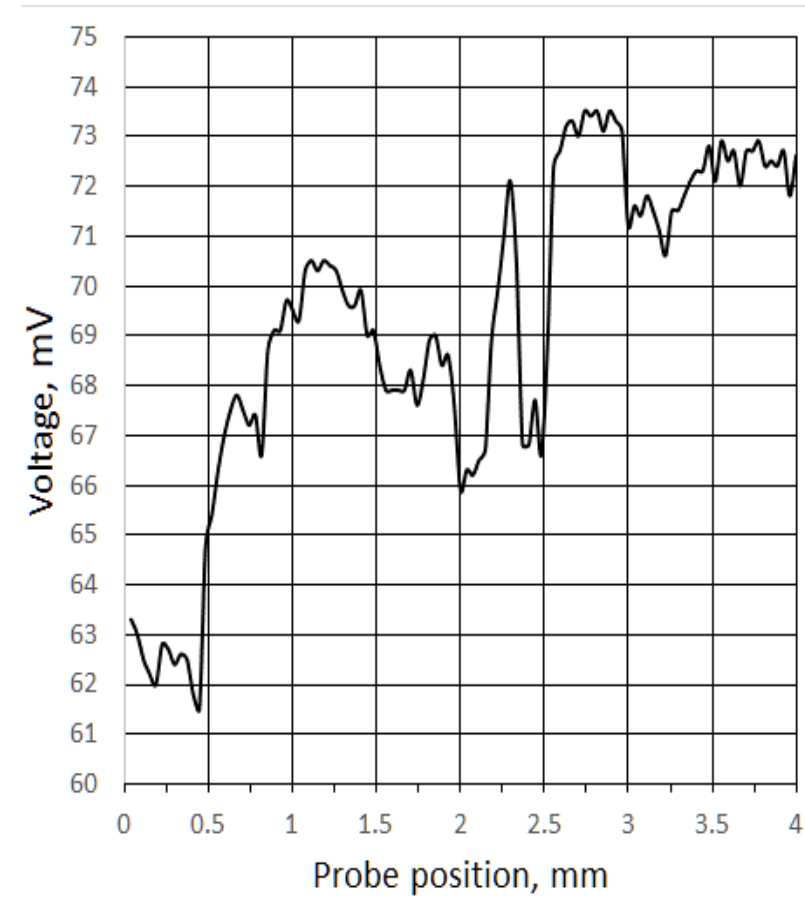

Fig. 4. A pattern observed when the probe moves along the layered medium with a defect. The frequency of the transducer is $7000 \mathrm{~Hz}$.

When operating frequency of the device is beyond the given limits, the results of the measurements will be distorted by amplitude fluctuations caused by microcracks on the 
surface of the sample or by the decreasing of field localization inside the layered structure. Fig. 4 shows the measurement results of the sample at a frequency of $7000 \mathrm{~Hz}$. As seen from the graph, the amplitude changes, caused, in this case, by the microcracks on the sample surface, are much higher than the amplitude changes, caused by the defect directly.

\section{B. Scanning the structure steel-paper}

The experiment was carried out with two samples of electrotechnical steel, sample No. 1 - 1212 steel, sample No. 2 - 3414 steel. Samples were located at a distance of $1 \mathrm{~cm}$, a dielectric (paper) was placed between samples. The signal applied to ECT energizing winding varied from 1000 to 10000 Hz. Voltage supplied to ECT measuring winding when it moved relative to the initial point of scanning was applied as a measurable parameter. The scanning was carried out continuously (ECT was moving above samples at a constant speed of $1 \mathrm{~mm} / \mathrm{s}$ ) and discretely (ECT was changing its position at intervals of $0.1 \mathrm{~mm}$, measurement at each interval was performed for $0.5 \mathrm{~s}$ ).

The curve of introduced voltage amplitude as a function of sensor location was constructed to study in details the introduced voltage drop at the ferromagnetic-dielectric interface. This curve allows to demonstrate behavior of the introduced voltage amplitude when eddy-current transducer is moving above steel-dielectric interface. Scanning started at a distance of $5 \mathrm{~mm}$ from the edge of the first sample to a point that corresponds to a distance of $5 \mathrm{~mm}$ from the edge of the second sample. When scanning, observing of ferromagneticdielectric interface is carrying out with qualitative binding to distance to and after the interface of the object that is under research. Curve of introduced voltage amplitude as a function of sensor location indicates effects occurring at the metaldielectric interface. Experimental results are shown in Fig. 5.

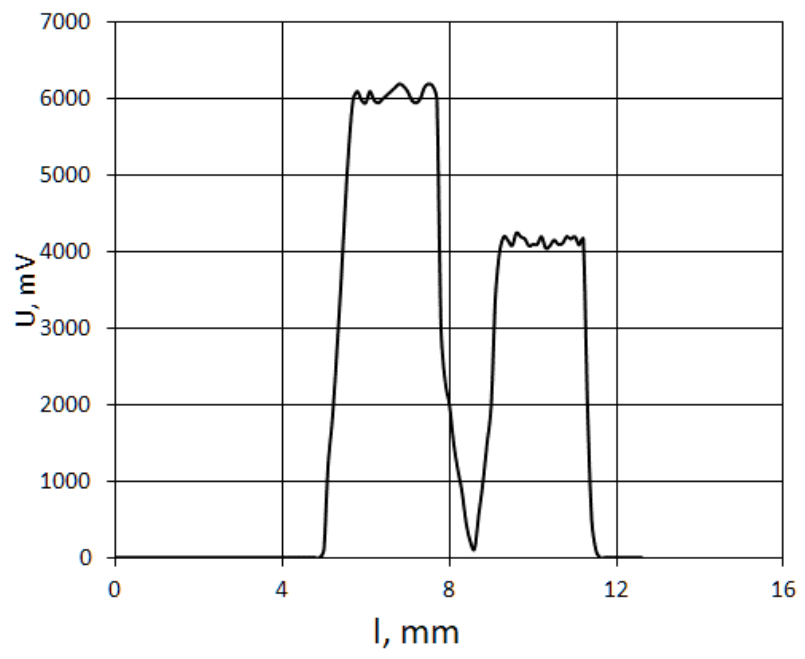

Fig. 5. Dependence curve of induced voltage on the gage position while continuous scanning at $1000 \mathrm{~Hz}, 1$ - gage position, U - introduced voltage

Two different peaks of introduced voltage on the curve correspond to two different types of electric steel with different permittivity. The curve shows that when the sensor moves from steel to dielectric, introduced voltage is suddenly dropping from the value of $6000 \mathrm{mV}$ to $2000 \mathrm{mV}$, following which tends to zero gradually. To study in details the introduced voltage changing at the ferromagnetic-dielectric interface, the digital scanning of this interface was executed and the relevant curve of introduced voltage amplitude as a function of transducer location was constructed (Fig. 6). This experiment involved the local digital scanning of the objects that are under research: in this respect, the scan shots were performed at points situated at a distance of $0.1 \mathrm{~mm}$ from one another.

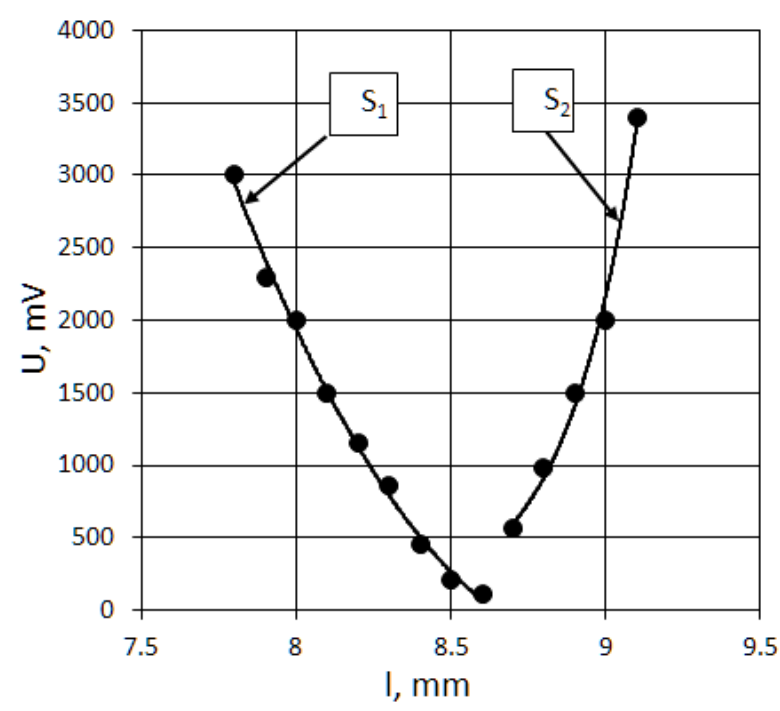

Fig. 6. Dependence curve of induced voltage amplitude on the gage position while discrete scanning at $1000 \mathrm{~Hz}, \mathrm{l}$ - gage position, $\mathrm{U}$ - introduced voltage, $S_{1}-$ area $1, S_{2}-$ area 2 .

This experiment allows one to draw the following conclusion: at the steel-dielectric interface, response from ferromagnetic is not suddenly drops to zero but decreases in quadratic dependence (Area 1). At that, the minimum value of signal amplitude is $50 \mathrm{mV}$. Increasing of signal amplitude progressively as approaching the second steel sample occurs in dependence that is close to exponential (Area 2).

The resulting dependence is due to the residual voltage in ECT measuring windings. Closed power lines are exuded in electrical steel field. Due to this the electromagnetic field generated by eddy currents and preventing the self-inductance in measuring winding will not strongly affect on inserted voltage. Thus response value does not decrease to zero because of the influence of the ferromagnet on ECT.

The influence of a ferromagnet on ECT may be explained by the presence of steel self-magnetic field, which closes at ECT even at a relatively large distance.

In this case voltage value at measuring winding is much less than voltage applied to winding when ECT is placed directly above steel. It is seen on the diagram that voltage continues to decrease as the distance between ECT and steel increases. As we approach to the second steel sample, voltage 
amplitude increases. This increase occurs exponentially, which is caused by the addition of magnetic fields of the first and second samples.

To estimate the influence of ECT signal frequency on measurement results, the dependence of the amplitude of the applied voltage on the position of the sensor at a frequency of $10000 \mathrm{~Hz}$ was developed. The results of the experiment are shown in Fig. 7.

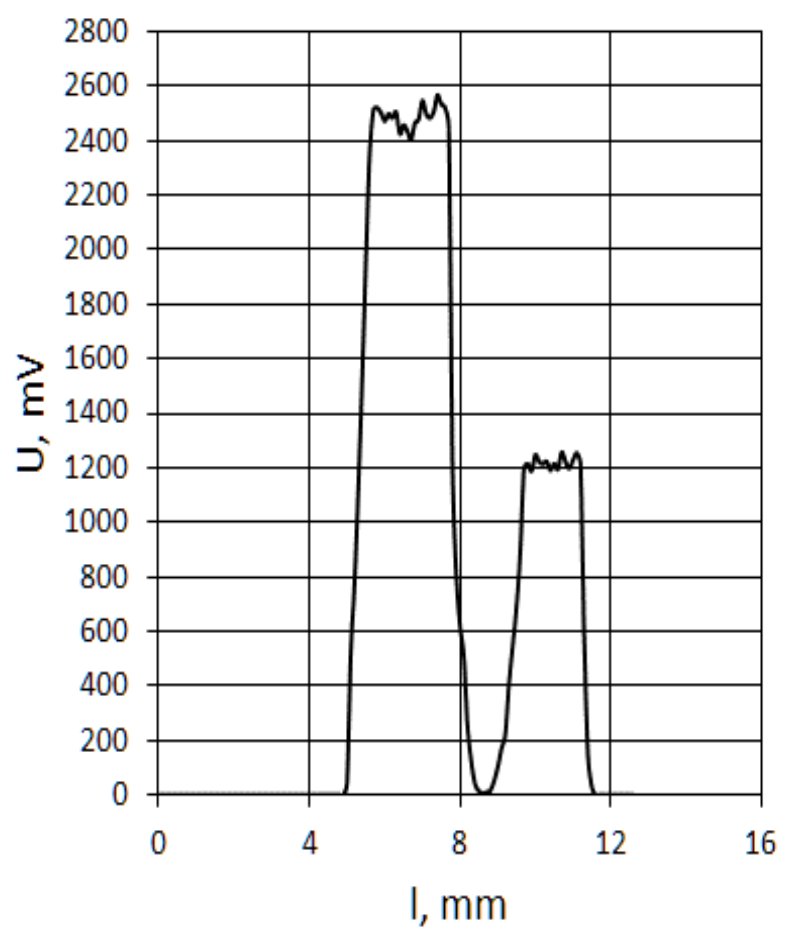

Fig. 7. Dependence curve of induced voltage on the gage position while continuous scanning at $10000 \mathrm{~Hz}, 1$ - gage position, U - introduced voltage

The qualitative nature of the dependence of ECT voltage on its position has practically not changed. Two peaks of the applied voltage correspond to the two samples under study. Sudden drop in voltage corresponds to area of dielectric location, that separated the samples. However, the rate of voltage drop has significantly changed when moving away from the first sample. In addition, the minimum voltage (5 $\mathrm{mV}$ ) has changed, that corresponds to the moment when sensor was passing above dielectric. These changes are due to the greatly increased magnetic losses of steel associated with an increase in the frequency of the current to $10000 \mathrm{~Hz}$. At a given frequency, the magnetic field of the steel has a significantly lower influence on applied voltage than in the case corresponding to $1000 \mathrm{~Hz}$. It is shown in Fig. 8 that drop in applied voltage in area 1 occurs exponentially, unlike Fig. 6. Voltage increase in area 2 also occurs exponentially.

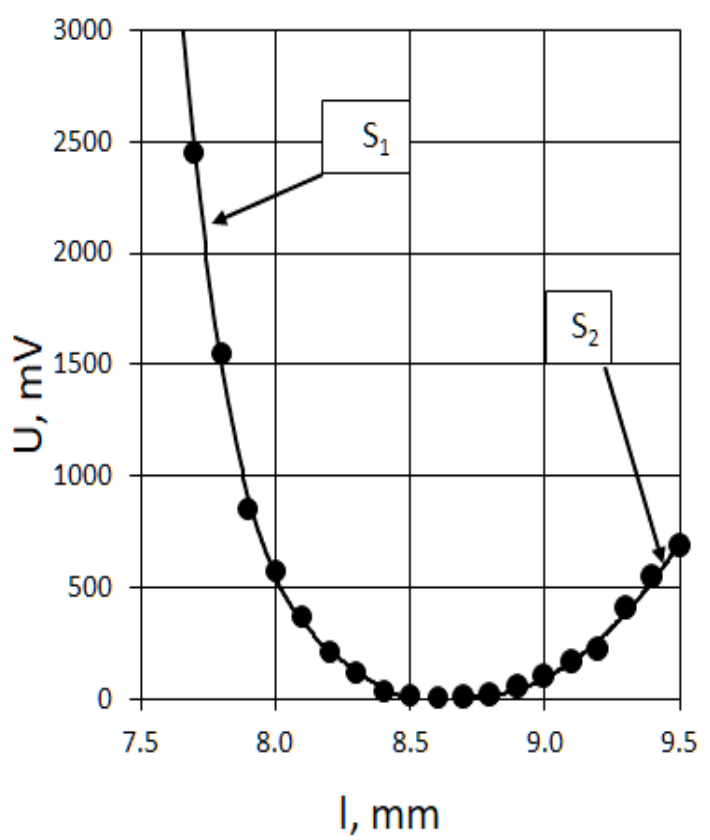

Fig. 8. Dependence curve of induced voltage amplitude on the gage position while discrete scanning at $10000 \mathrm{~Hz}, 1$ - gage position, $\mathrm{U}$ - introduced voltage, $S_{1}-$ area $1, S_{2}-$ area 2 .

In the course of the final experiment, the influence of the gap between transducer and electrical steel on voltage applied to ECT measuring winding was studied. In the course of the experiment, sample No. 1 was subject to a digital scanning. Measurements were made at a current frequency of $1000 \mathrm{~Hz}$, the gap increased at an interval of $0.1 \mathrm{~mm}$. The results of the experiment are shown in Fig. 9.

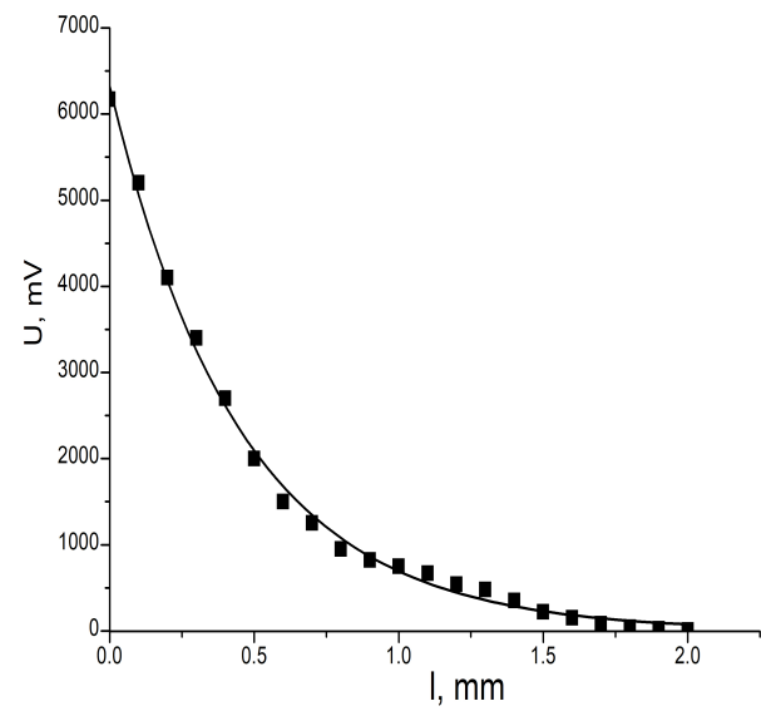

Fig. 9. Dependence curve of induced voltage on gap while discrete scanning at $1000 \mathrm{~Hz}, 1$ - object distance, $\mathrm{U}$ - introduced voltage

The presented dependence shows that when the sensor was removed from the steel, voltage decreased exponentially. This is due to the inadequate depth of penetration of an 
electromagnetic field generated by ECT exciting winding in steel when it is placed at a large distance from the object of control. In addition, influence of magnetic lines of steel on measuring winding becomes noticeably weaker with increasing of a distance.

\section{CONCLUSIONS}

Based on a eddy current transducer (ECT), a probe has been designed to research metal-dielectric structures. The transducer was tested on the layered structure consisting of paper and aluminum layers with a thickness of $100 \mu \mathrm{m}$ each in which the model defect was placed. Right up to the depth of the defect, equal to $1400 \mu \mathrm{m}$, there is a clear dependence of the response of transducer, on the position of the transducer over the defect. By fixing change in amplitude response in the converter caused by a defect, it is possible to change the frequency of the current in the exciting winding so that the eddy-currents are concentrated in the layers of composite placed above the defect. The solution of the inverse problem allows to determine the depth of the defect.

Also, we applied the developed measuring system to examine the electric steel parameters. We determined that mathematical functions were obtained to describe variation of introduced voltage while scanning steel to dielectric transitions at various ECC signal frequencies and to describe variation of introduced voltage with varied gap. This allows us to prospectively use the amplitude control method of such object class for exact local measurements of the thickness of conductive and non-conductive coatings as well as of other objects. The investigation also proves that the measurement system is appropriate for thickness measurement and for studying structural properties of steel.

The presented eddy-current transducer for structural materials investigation can be used for diagnostic and control of critical joints and structural machine elements used in electrical engineering.

\section{References}

[1] L. Barbato, N. Poulakis, A. Tamburrino, T. Theodoulidis, S. Ventre, "Solution and extension of a new benchmark problem for eddy current nondestructive testing", IEEE Trans. Magn, vol. 51(7), pp. 1-7, July 2015.

[2] R. Mak-Master, Non-destructive testing: reference book, ENERGIYA, Moscow, 1965.

[3] A. Katunin, M. Danczak, P. Kostka, "Automated identification and classification of internal defects in composite structures using computed tomography and 3D wavelet analysis", Arch. of Civ. Mech. Eng, vol. 15(2), pp.436-448, March 2015.

[4] W. J. Staszewski, S. Mahzan, R. Traynor, "Health monitoring of aerospace composite structures - Active and passive approach", Comp. Sci. Tech, vol. 69(11-12), pp. 1678-1685, 2009.

[5] A. Katunin, K. Dragan, "Damage identification in aircraft composite structures: A case study using various nondestructive testing techniques", Comp. structur, vol. 127, pp. 1-9, April 2015

[6] C. Maierhofer, P. Myrach, "Characterizing damage in CFRP structures using flash thermography in reflection and transmission configurations", Comp. Part B: Eng, vol. 57, pp. 35-46, October 2014.

[7] T. H. Loutas, A. Panopoulou, "Intelligent health monitoring of aerospace composite structures based on dynamic strain measurements", Exp. Sys. with Applic, vol. 39(9), pp. 8412-8422, March 2012.
[8] D. D. Rizos, S. D. Fassois, "Vibration-based skin damage statistical detection and restoration assessment in a stiffened aircraft panel", Mech. Sys.Signal Proc, vol. 22(2), pp. 315-337, December 2008.

[9] C. Meola, "Infrared thermography to evaluate impact damage in glass/epoxy with manufacturing defects”, Int. Journal Imp. Eng, vol. 67, pp. 1-11, October 2014.

[10] R. Růžek, R. Lohonka, "Ultrasonic C-Scan and shearography NDI techniques evaluation of impact defects identification", NDT \& E Int, vol. 39(2), pp. 132-142, June 2006.

[11] D. J. Bull, S. M. Spearing, "Three-dimensional assessment of low velocity impact damage in particle toughened composite laminates using micro-focus X-ray computed tomography and synchrotron radiation laminography”, Comp. Part A: Appl. Sci. Manuf, vol. 52(1), pp. 62-69, December 2013.

[12] S. Grondel, "Health monitoring of a composite wingbox structure", Ultrasonics, vol. 42(1-9), pp. 819-824, September 2004.

[13] U. Polimeno, M. Meo, "Detecting barely visible impact damage detection on aircraft composites structures", Comp. struct, vol. 91(4), pp. 398-402, September 2009.

[14] T. H. Loutas, "Panopoulou, Intelligent health monitoring of aerospace composite structures based on dynamic strain measurements", Expert Sys. with App, vol. 39(9), pp. 8412-8422, May 2012.

[15] K. Warnemuende, Amplitude modulated acousto-ultrasonic nondestructive testing: Damage evaluation in concrete, Ph.D. Wayne State University, Ann Arbor, 2006.

[16] I. Oguma, R. Goto, "Ultrasonic inspection of an internal flaw in a ferromagnetic specimen using angle beam EMATs", Przeglad Elektrotechniczny, vol. 88(7B), pp. 78-81, December 2012.

[17] K. Koyama, H. Hoshikawa, "Eddy Current Nondestructive Testing for Carbon Fiber- Reinforced Composites", Jour. Press. Vess. Tech, vol. 135(4), pp. 041501-041501, February 2013.

[18] S.-H. Yang, K.-B. Kim, "Non-contact detection of impact damage in CFRP composites using millimeter-wave reflection and considering carbon fiber direction", NDT \& E Int, vol. 57(1), pp. 45-51, June 2013.

[19] I. Tomáš, "Nondestructive indication of fatigue damage and residual lifetime in ferromagnetic construction materials", Meas. Sci. and Tech, vol. 25, pp. 1-10, May 2014.

[20] A.V. Egorov, S.V. Kucheryavskiy, V.V. Polyakov, "Resolution of effects in multi-frequency eddy current data for reliable diagnostics of conductive materials", Chemom. and Intell. Labor. Syst, vol. 160, pp. 812, March 2017.

[21] R. Ghoni, M. Dollah, "Defect characterization based on eddy current technique: Technical review", Adv. in Mech. Eng, vol. 6, pp. 1-11, August 2015.

[22] [D. Rifai, A. N. Abdalla, N. Khamsah, M. Aizat, M. Fadzli, "Subsurface defects evaluation using eddy current testing", Indian Journal of Science \& Technology, vol. 9(9), pp. 1-7, April 2016.

[23] J.M. Szlagowska-Spychalska, M.M. Spychalski, “A novel approach for measuring of thickness of induction hardened layers based on the eddy current method and the finite element modeling", NDT E. Int, vol. 54, pp. 56-62, May 2013.

[24] [G. Almeida, J. Gonzalez, L. Rosado, "Advances in NDT and materials characterization by eddy currents", Procedia CIRP, vol. 7, pp. 359-364, June 2013.

[25] D. Rifai, A.N. Abdalla, "Defect signal analysis for nondestructive testing”, ARPN J. Eng. Appl. Sci, vol. 11, pp. 1-14, August 2015.

[26] [A. N. Abdalla, R. Razali, "An eddy current testing platform system for pipe defect inspection based on an optimized eddy current technique probe design", Sensors (Basel), vol. 17(3), pp. 1-24, May 2017.

[27] S. Dmitriev, A. Ishkov, V. Malikov, and A. Sagalakov, "Subminiature eddy current transducers for studying metal-dielectric junctions", Instr. and Exp. Tech, vol. 57(6), pp. 751-754, June 2014.

[28] S.F. Dmitriev, V.N. Malikov, A.M. Sagalakov, A.V. Ishkov, A.O. Katasonov, "Subminiature eddy-current transducers for conductive materials and layered composites research", Adv.Intell. Sys. Comp, vol. 692, pp. 655-665, December 2017. 